\title{
CARACTERIZACIÓN DEL CONSUMIDOR UNIVERSITARIO: UN ESTUDIO ETNOGRÁFICO
}

Margarita Contreras Cuenta Universidad del Norte Colombia

Yolmis Nicolás Rojano Alvarado Universidad de La Guajira Colombia

Diego Cardona Arbeláez Universidad Libre de Colombia Colombia 
Panorama Económico, Vol. 27 - No. 2 (Abril - Junio de 2019), pp. 559-574

\author{
Margarita Contreras Cuenta \\ Yolmis Nicolás Rojano Alvarado \\ Diego Cardona Arbeláez \\ JEL: M21, M30, M39
}

\title{
Caracterización del consumidor universitario: un estudio etnográfico
}

\section{Resumen}

El presente documento analiza las características y hábitos de compras de los consumidores de la tienda universitaria en una reconocida universidad del caribe colombiano. Se busca descubrir las costumbres, los valores, las tradiciones de los consumidores de la comunidad universitaria y que conduzca al descubrimiento de los intereses, deseos y necesidades entre los individuos y sus hábitos de compras. La investigación se desarrolló a través del paradigma cualitativo, mediante el uso de una metodología etnográfica para la interpretación de datos. La investigación se desarrolló en cinco fases, inicialmente se efectuó una exploración de textos especializados que desarrollen el tema de la cultura de consumo, marketing y experiencias en tiendas universitarias; luego se realizaron observaciones con el fin de identificar los aspectos del consumidor relacionados con su cultura de consumo; se desarrollaron once (11) entrevistas a profundidad aplicadas a alumnos de las distintas facultades y cursos, profesores, funcionarios de la institución. Finalmente se realizó la transcripción de las entrevistas y se categorizaron de acuerdo con el propósito del estudio. El análisis de los resultados permite concluir la existencia de tres tipos de consumidores: consumidores por línea, consumidores por fidelidad y consumidores por frecuencia.

Palabras clave: Consumidor, Tienda Universitaria, Etnografía

\section{Caractérisation du consommateur universitaire: une étude ethnographique}

\begin{abstract}
Résumé
Ce document analyse les caractéristiques et les habitudes d'achat des consommateurs du magasin universitaire d'une université renommée des Caraïbes colombiennes. Il vise à découvrir les coutumes, les valeurs, les traditions des consommateurs dans la communauté universitaire et à conduire à la découverte des intérêts, des désirs et des besoins des individus et de leurs habitudes d'achat. La recherche a été développée à travers le paradigme qualitatif, à travers l'utilisation d'une méthodologie ethnographique pour l'interprétation des données. La recherche s'est déroulée en cinq phases, dans un premier temps une exploration de textes spécialisés développant le sujet de la culture de consommation, du marketing et des expériences dans les magasins universitaires a été réalisée; Des observations ont ensuite été faites afin d'identifier les aspects du consommateur liés à sa culture de consommation; onze (11) entretiens approfondis ont été réalisés, appliqués aux étudiants des différentes facultés et cours, aux professeurs et aux responsables de l'établissement. Enfin, les entretiens ont été transcrits et classés en fonction de l'objectif de l'étude. L'analyse des résultats permet de conclure à l'existence de trois types de consommateurs: les consommateurs par ligne, les consommateurs par fidélité et les consommateurs par fréquence.
\end{abstract}

Mots clés: Consommateur, Boutique universitaire, Ethnographie

\section{Characterization of the university consumer: an ethnographic study}

\begin{abstract}
This document analyzes the characteristics and purchasing habits of the consumers of the university store in a renowned university in the Colombian Caribbean. It seeks to discover the customs, values, traditions of consumers in the university community and to lead to the discovery of interests, desires and needs among individuals and their shopping habits. The research was developed through the qualitative paradigm, through the use of an ethnographic methodology for data interpretation. The research was carried out in five phases, initially an exploration of specialized texts that developed the subject of consumer culture, marketing and experiences in university stores was carried out; Then observations were made in order to identify the aspects of the consumer related to their consumption culture; eleven (11) in-depth interviews were carried out, applied to students from the different faculties and courses, professors, and officials of the institution. Finally, the interviews were transcribed and were categorized according to the purpose of the study. The analysis of the results allows us to conclude the existence of three types of consumers: consumers by line, consumers by loyalty and consumers by frequency.
\end{abstract}

Keywords: Consumer, University Store, Ethnography 


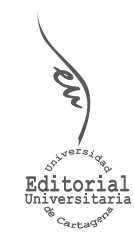

Artículo de Investigación

Caracterización del consumidor universitario: un estudio etnográfico

INFORMACIÓN DEL ARTÍCULO

Recepción del artículo: 19/10/2018

Concepto de evaluación: $26 / 11 / 2018$

Aceptación del artículo: 15/01/2019
Margarita Contreras Cuenta Universidad del Norte, Colombia

Yolmis Nicolás Rojano Alvarado Universidad de La Guajira, Colombia

Diego A. Cardona Arbeláez* Universidad Libre de Colombia, Colombia

\section{INTRODUCCIÓN}

En la historia de las universidades del mundo con relación a las tiendas universitarias, se demuestra que este tipo de locales comerciales han sido participe del desarrollo e identidad corporativa de las instituciones educativas, y en su mayoría se apoyan en las estrategias de marketing, las actividades de bienestar con los estudiantes distinguiéndose el deporte en gran número de productos que comercializan. La investigación de Serrano y Goyeneche (2008) denominada "Creación de una Tienda Universitaria con productos marca UIS" describe algunas tiendas existentes en Europa, como las de las universidades de Sevilla, Vigo en España y la de Mantua, en Italia, las cuales poseen: a) de igual condición se destacan por ofrecer productos institucionales, b) la tienda de Vigo tiene una construcción vanguardista y brinda además un lugar de encuentro para universitarios y profesores, c) la de Mantua tiene como misión la historia y tradiciones que la hacen famosa. Y además, en el mismo trabajo se enfatiza en las tiendas de los Estados Unidos como las de Universidad de Stanford y la de la Universidad de Washington, ambas muy representativas de las tendencias universitarias de la cultura norteamericana, relacionando los gustos y expresiones que lideran con el deporte interinstitucional del país siendo el punto inicial para proponer al consumidor todo tipo de productos de accesorios, ropa, calzado, suvenires etc., con logos de los equipos y hasta mascotas que los representan.

En Colombia son pocas las tiendas universitarias y entre las que se distinguen la Tienda Universitaria de la Universidad de Antioquia, la Universidad de EAFIT que se ocupan fomentar el sentido de pertenencia para con todos los actores de las instituciones, y la tienda de la Universidad Javeriana que se distingue por la comercialización de textos y ejemplares de publicación. En la tienda de la Universidad de Antioquia describe en su portar virtual (ver Imagen 2) que tiene varios objetivos como: 1) Satisfacer las 
necesidades del cuerpo universitario, docente y administrativo de la Universidad, 2) Mantener una alta calidad en sus productos, con precios competitivos, y 3) Conservar un proceso administrativo de mejora continua en el servicio, buscando que se tenga siempre ingresos y utilidades crecientes; ajustándose a las necesidades de los actores del campus universitario pero muy discreta en un posicionamiento generalizado de su marca institucional en los productos que ofrece.

En la Región Caribe Colombiana, la Universidad insignia es la Universidad del Norte, cuenta con una tienda universitaria con características poco institucionales, y su origen inicia en 1998, cuando la Universidad cedió un espacio físico en una área pequeña de uno de los bloques de uso académico, entregada en administración a la Asociación de Egresados quienes deberían aportar a través de la tienda un servicio aliado a la imagen de la Universidad del Norte y obtener recursos que apoyaran sus actividades como un grupo reconocido.

Enelpresentearticuloserealizóun análisis sobrela caracterización delos consumidores universitarios que frecuentan los servicios de una importante universidad del Caribe colombiano con ubicación en el distrito industrial de Barranquilla - Colombia, con el objeto de conocer el tipo de producto que estos demandan.

\section{MARCO TEÓRICO}

\section{Cultura}

El estudio de los mercados se traduce a la comprensión más cercana de los individuos, representados en sus acciones, la relación con su medio y sus comportamientos, resultando de la suma entre unos y otros para brindar las más sutiles características que pueden ser tomadas por empresarios, emprendedores y/o líderes de negocios en sus planes de marketing. Es así, como refiere Páramo (2004) que "la complejidad propia de la posmodernidad en la que el mundo de los negocios se ha desenvuelto, ha impuesto retos cada vez más indescifrables a quienes desde la empresa han debido atender un mercado más exigente y con mayores opciones para ser satisfecho".

La cultura está dada en el sentido más cercano al comportamiento de los individuos, se diferencia dependiendo los grupos sociales, el desarrollo de estos en su historia y evolución del contexto (elementos materiales e inmateriales). Páramo, Domingo y Ramírez (2007) aseguran que:

"...es innegable, la cultura es una idea que ha llegado sin pedirle consentimiento a nadie, despertando zozobras e inquietudes, zambulléndose entre el mundo consciente o inconsciente de los miembros de un mismo grupo social quienes, casi sin percatarse, la llevan consigo transportando sus propios patrones de pensamiento, sentimiento y potencial forma de actuación; todos ellos aprendidos a lo largo de sus propias existencias." (p.5).

\section{La cultura de consumo}

De acuerdo Kozinets (como se citó en Martínez, 2009), este término de:

"la Cultura de Consumo es utilizado para definir un particular sistema interconectado de imágenes, textos, y objetos producidos comercialmente que ciertos grupos usan, a través de la construcción de superpuestas e incluso 
conflictivas prácticas, identidades y significados, para darle sentido colectivo a sus ambientes y para orientar la experiencia y la vida de sus miembros".

Indudablemente, el mundo moderno ha venido dejando de lado la idea de considerar los bienes como elementos meramente útiles, que tienen valor de uso y valor de cambio para adoptar una visión individualizaste, como señala Álvarez (2014) al "relacionar el consumo con la vivencia de placeres emocionales y estéticos de carácter personal". Pero aún desde la perspectiva de la cultura, Schouten \& McAlexander (1995) describen que

“...existen posiciones diversas de interpretación. Por una parte, la cultura de consumo se entiende como subcultura, pero, por otra parte, también puede ser considerada como un factor que va más allá de las normas, los valores y principios de una cultura determinada. La cultura de consumo siempre hace referencia a un subgrupo específico dentro de una sociedad que comparte normas, creencias y valores en relación con una clase de producto, una marca o de una actividad de consumo".

Este subgrupo incluye, además que "una cultura de consumo se vuelve real en la medida en que las personas se identifican con ciertos objetos o actividades de consumo, a su vez, permite identificarse con otros individuos. Su estructura, la cual rige las interacciones sociales creadas dentro de ella, es un reflejo directo del compromiso de los individuos de acuerdo con los deberes adquiridos" (Martínez, 2009).

La cultura de consumo es pues, una forma social de interpretar la relación entre el mercado y los objetos y por ello, el consumo se entiende a su vez como un fenómeno social que tiene que ver con los distintos aspectos de la vida de los individuos. Páramo, Ramírez y Martínez, (2007) dicen que "el consumo es concebido como como la conjugación y expresión de una serie de circunstancias propias del mundo moderno particularmente complejas y estrechamente relacionadas con el acto y la acción de adquisición, apropiación y uso de productos - bienes, servicios e ideas-, se haya convertido en la columna vertebral de los actuales procesos del marketing moderno".

Dentro de la perspectiva antropológica, McCracken deja la reflexión sobre considerar que el consumo es esencialmente un fenómeno cultural cargado de significado, aunque ciertamente puede cambiar de acuerdo con la moda y la publicidad. McCracken (1990) tiene claro que los individuos son un producto de su cultura y de sus grupos sociales y por esta razón toda su actuación social está mediada por comportamientos rituales. Sin embargo, se debe señalar que la era virtual o digital ha ganado espacios y ha cambiado conducta del consumidor, de tal suerte que ha emergido tendencias como el marketing digital y sus distintas perspectivas (Fierro, 2017).

La experiencia del consumo es asociada con la marca, y la construcción de la marca se genera a partir de los momentos de interacción con otros en un contexto específico que reafirman los valores que se desarrollan alrededor de ésta. Por tal motivo es importante reconocer como los consumidores asumen, viven e idealizan su experiencia, de tal suerte, que brinde información valiosa para la gestión de la marca (Del Rio, 2017).

Por tal motivo cobra importancia poder construir una imagen de marca fuerte, porque ofrece claras ventajas que generan competitividad, siempre y cuando dichas 
acciones permitan generar diferenciación, identificación y seducir el imaginario de los consumidores sobre la marca (Del Rio, 2017).

De acuerdo con los estudios adelantados por la CEPAL (2000) consideran que "Hoy en día los jóvenes tienen un amplio acceso a los servicios de educación, trabajo, salud y vivienda, así como al consumo físico y simbólico del espacio y los bienes y servicios que se ofertan. La publicidad de bienes y servicios que impulsa al consumismo está encaminada a la estimulación del deseo y la necesidad por la adquisición de estos, hasta el punto de generar una sensación de fracaso si no pueden obtenerse, lo cual repercute en la baja de la representación que tienen de sí mismos".

\section{La Tienda Universitaria}

En el mundo las tiendas universitarias han tomado una importante representatividad, como parte de la venta de servicio en el sector educativo de educación superior, estableciendo una fuerte relación entre la actividad deportiva de la institución y el gusto de los jóvenes por esta actividad un ejemplo de ello es la Stanford University, se promociona la marca deportiva Stanford cardinal, utilizada por los equipos deportivos de nivel competitivo de la Universidad Cardinal que participan en la National Collegiate Athletic Association (NCAA) conformada por los menos por 1200 instituciones de los Estados Unidos de Norte América.

Se aprecia en la (NCAA), una tienda virtual exclusiva para admiradores de las escuelas de deportivas, a pesar de que a sus inicios este espacio en la web era utilizado para tener una comercialización de libros; demostrando la fuerza cultural que penetro y posiciono en esta universidad la marcada identidad deportiva que hace parte de la comunidad. Es así, como las empresas y grandes marcas en EE. UU tomen la costumbre comercial de apoyar a las tiendas universitarias patrocinando sus equipos y en relación comercializar sus productos que ellos puedan fabricar resaltando el nombre de la institución (Serrano y Goyeneche, 2008).

En Colombia, son pocas las tiendas universitarias y entre las que se distinguen la Tienda Universitaria de la Universidad de Antioquia, la Universidad de EAFIT que se ocupan fomentar el sentido de pertenencia para con todos los actores de las instituciones, y la tienda de la Universidad Javeriana que se distingue por la comercialización de textos y ejemplares de publicación. En la tienda de la Universidad de Antioquia describe en su portal virtual que tiene varios objetivos como: 1) Satisfacer las necesidades del cuerpo universitario, docente y administrativo de la Universidad, 2) Mantener una alta calidad en sus productos, con precios competitivos, y 3) Conservar un proceso administrativo de mejora continua en el servicio, buscando que se tenga siempre ingresos y utilidades crecientes; ajustándose a las necesidades de los actores del campus universitario pero muy discreta en un posicionamiento generalizado de su marca institucional en los productos que ofrece.

En la universidad del Norte, se tomó como antecedente el estudio sobre Farmatodo realizado en Bogotá en 2009, donde se mostró la existencia de nueve categorías de productos, que en orden de importancia quedaron así: En primer lugar: Dulces, OTC, Belleza, Cuidado Personal, Dieta/Fitness. En segundo lugar: Productos para el hogar, Salud Sexual y Temporadas, y como de menor priorización: bebés/niños, para ser un ejemplo en la tienda universitaria. Asimismo, en cuanto a la presentación física del almacén, los participantes, proponen características como: iluminación con acentos/ 
matizonas (flexible, Tipo L'Occitane /Cálido, Estilo moderno, Piso liso (un solo color o madera), con olor, Pisos claros, con vitrinas - solo vidrio, Slimdoor, Vintage, Música ambiental, Blancos/beige, Cajas autoasistidas, Porta precios y señalización versátil, Accesorios comunicación, Mobiliarios con depósito. Poca profundidad, Smart layout. Distribución inteligente, Sensación de amplitud/altura/pasillo, Distribución inteligente donde no se obstaculice el ingreso y el camino del cliente con las cajas y la salida de este.

\section{MARCO METODOLÓGICO}

Para el desarrollo del presente articulo se realizó una investigación con el uso del paradigma cualitativo, con utilización del método etnográfico y el apoyo de la antropología para la interpretación de los datos. Según Méndez (como se citó en Páramo, Contreras y Arenas 2010) afirma que la investigación en las ciencias sociales se ocupa de la descripción de las características que identifican los diferentes elementos y componentes, y su interrelación.

En primer momento, se efectuó una exploración de textos especializados que desarrollen el tema de la cultura de consumo, puntos de venta, marketing y experiencias en tiendas universitarias, utilizando diversas fuentes que incluyen artículos científicos, libros, revistas, páginas de internet; se desarrollo un trabajo de campo en la institución Universitaria objeto de análisis, se realizaron observaciones pertinentes y se entró en el contacto con aquellos aspectos del consumidor que nos permitieron conocer de su cultura, hasta identificar sus intereses, valores, costumbres los potenciales consumidores de la tienda universitaria; posteriormente, se realizo una observación por cinco días esparcidos en medio de la investigación en una (1) hora promedio en cada día la observación de los comportamientos de los participantes en la tienda Universitaria, logrando extraer conocimiento y comparar con el avance de los resultados de la investigación, posteriormente se desarrollaron once (11) entrevistas a profundidad, la selección de informantes se hace a través del método no probabilístico que algunos autores denominan "muestreo intencional u opinático" (Goetz y Le Compte, Citados por Galindo 1998). Para ello se establecieron los siguientes criterios: Alumnos de las distintas facultades y cursos, profesores y funcionarios de la institución.

Finalmente, se realizó la transcripción de las entrevistas y se categorizarán de acuerdo con las temáticas a investigar, cada entrevista se analizó y el proceso de estudio de cada una de ellas se apoyó para la interpretación de estas y de la información observada sobre la tienda universitaria, como resultado de este análisis resultaron tres matrices, en las cuales se muestra el proceso creativo de la presentación de los resultados.

\section{RESULTADOS}

Se realizó la transcripción de cada entrevista, el surgimiento de categorías emergentes, permitió su organización por temáticas con respecto a cada objetivo. En consecuencia, cada entrevista se analizó, llevando a la clasificación trasversal (matrices) cada una de las impresiones de las unidades de análisis. En este sentido, la construcción de tres matrices, fueron necesarias, en cada una de ellas se muestra el proceso creativo de la presentación de los resultados:

\section{a. Matriz de Análisis por Actores}

Se desarrolló realizando un análisis con relación al objetivo planteado en el 
estudio, por ello, se incluyeron las siguientes columnas que sirvieron para dar como resultado las características generales.

\section{b. Matriz de Características}

Una vez identificados los hallazgos y relacionados con las características encontradas se procedió a utilizar una matriz para agrupar de manera organizada las características encontradas y la explicación de estas.

\section{c. Matriz de Resumen por Temas}

Es una matriz final donde surge la base de los hallazgos de la investigación y se realiza la asociación teórica.

Se encontraron tres grandes tipos de consumidores: consumidores en linea (consumidor de artículos personales, consumidor de comestibles y drogas, consumidor de productos típicos); consumidores por fidelización (consumidor por identidad académica, consumidor por variedad, consumidor de servicios y asesorías, consumidor de promocional); consumidores por frecuencia (consumidor habitual, consumidor ocasional). Los cuales se segregan de acuerdo con las características particulares:

\section{Consumidores por líneas}

Se refiere a los consumidores que relacionan sus compras con el tipo de productos que desean adquirir. Los productos según su línea son aquellos que se destinan a usos esencialmente semejantes y que poseen características físicas muy parecidas. Hernández y Viveros (2012) dicen que "una línea de productos es un grupo de artículos que están estrechamente relacionados, ya sea porque se producen o comercializan de la misma manera, o porque satisfacen una clase de necesidad o se usan conjuntamente"

"A mí me gusta mucho la bisutería y yo diría que pega mucho porque allá hay blusitas y no sé si hay zapatos o sandalitas, pero es algo que a nosotras nos gusta tener algún accesorio.[...] Pues en general, la ropa como casual, deportiva... Me gusta como te digo, el tema de la bisutería, los accesorios, arito". E2

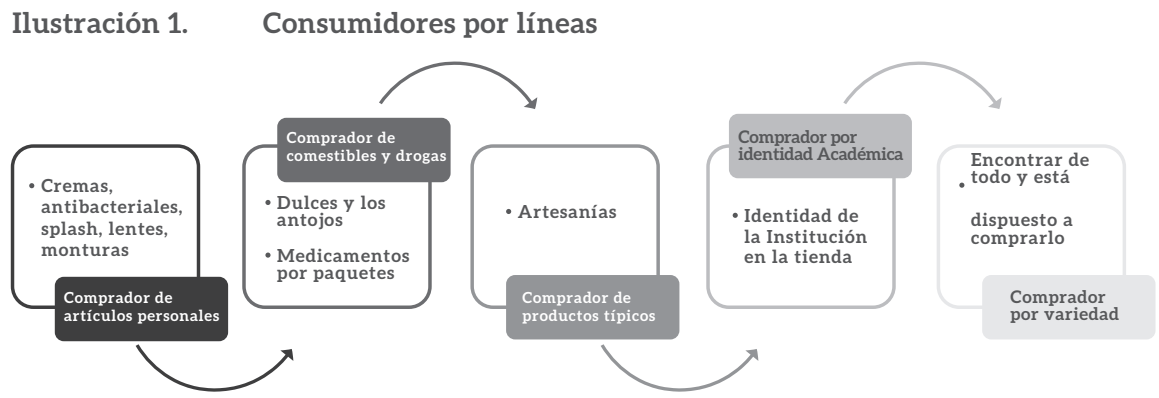

Fuente: Elaboración propia de los autores

\section{Consumidor de artículos personales.}

En la línea de artículos personales los consumidores de la tienda universitaria se interesan por cremas, antibacteriales y splash, por ejemplo. También se reporta el 
interés por lentes y monturas. Un entrevistado habla de los artículos deportivos relacionando los diversos deportes que se practican en la universidad y que ésta fomenta. Existe un énfasis en la ropa preferencia de ropa deportiva femenina, como de danza.

“[...] No sé porque normalmente compro las cosas que están acá. [....] De repente cremitas que no he visto y antibacteriales. He visto Splash, pero cremas y antibacteriales no". E05

Aquí también aparece el consumidor de tecnología y accesorios, como uno de los artículos que hacen parte de la vida diaria de los actores del campus universitario:

"Tecnología, IPad, IPhone. Me encanta Apple, entonces accesorios de esta marca, como forros, cargadores bacanos de tipo diferente, innovadores". F06

\section{Consumidor de comestibles y drogas:}

La línea de comestibles se genera por impulso y/o hábitos en los consumidores de la tienda universitaria, por ejemplo, como señala la Enciclopedia Larousse (2007) los comestibles se refieren a los dulces y los antojos, que se definen como el deseo, capricho y pasajero de algo En comestibles los dulces y antojos son los que más gustan.

Los consumidores de comestibles son uno de los más cercanos a ser clientes de la tienda universitaria, convirtiéndose los dulces uno de los productos más atractivos de la tienda, el que reporta mayor número de saturaciones en el estudio, prefiriendo una atención personalizada y no el uso de las máquinas dispensadoras; y aunque el surtido de dulces es bastante variado, los clientes están se ven interesados en incrementar esta línea de productos.

El consumidor por antojos "no soporta pasar frente a una tienda sin entrar a buscar algún producto que le satisfaga sus deseos. Aunque su consumo sea impredecible, existe una marcada tendencia a escoger aquello que lo atraiga de forma repentina que vea exhibido en la vitrina. En su rol de 'consentido' es un consumidor mimado y acostumbrado a recibir tratos especiales. Al relacionarse con la tienda, pide placer y diversión, pero sin extralimitarse. Demanda indulgencia con sus caprichitos y exige que se le dé gusto en todo" Páramo (2012)

"Me parece que tiene lo que uno necesita en cuanto a dulces, tiene muchas opciones, aunque a veces hay opciones más que uno espera".E07

Aunque la venta de medicinas requiera de unas condiciones y requisitos especiales, una vez cumplidos éstos, es posible que la tienda pueda poner a disposición de llamados "consumidores de medicamentos" sobre aquellos productos de mayor urgencia y de venta libre. El tipo de consumidores medicamentos son relacionados con la práctica al menudeo, se caracterizan por el deseo de encontrar en la tienda medicamentos que no sean por paquetes, para así, adecuarse a las realidades de los estudiantes y de los consumidores en general que no poseen un presupuesto de imprevistos para consumo de medicinas dentro de la universidad. Dentro de los gustos de consumidores de medicamentos se encuentran artículos que refieren a la limpieza del colon y vitaminas. 
"Yo diría que droguería como tal, farmacia, no. Algunos medicamentos de venta libre como Dolex , Redoxon".E02

“...las pastillas...todo lo venden en cajas, completo. Entonces si necesitas solo una y quizás cuando la vas a encontrar en el Centro Médico, tú no puedes comprar sólo una y tienes que comprar la caja entera. Así sea que cuando llegues a tu casa puedas ir a la farmacia y comprar la caja mucho más barata o comprar el remedio que en verdad necesitas" E07.

\section{Consumidor de productos tí picos:}

Según a Caldentey y Gómez (1996), "un producto es típico cuando se halla ligado de manera especial a un territorio y culturalmente a unas costumbres o modo que le diferencian de otros productos. En la entrevista se habla también de artesanías".

El consumidor de producto típico tiene la expectativa de conocer y/o identificarse con la cultura que representa el artículo, posee el imaginario de compartir e incluir a nuevas personas dentro del grupo social que los produce, y además valorar su artesanía, desde este concepto los consumidores plasman la compatibilidad de consumir este tipo de artículos. Asimismo, Polanyi (como se citó en Jaimovich, 2015) piensa que las sociedades modernas se forman a través de la transición de una red de intercambios recíprocos comunitarias a las interacciones del mercado institucionalizados.

“...se promocionan esos elementos o cosas típicas y encuentran allá a los estudiantes para que les hablen de su región".E01

Otro entrevistado señala que

“...hay muchas personas extranjeras y sería bueno que conocieran la cultura, no simplemente de Barranquilla, sino también de otras partes del país, porque se supone que vienen a conocer...Por decir, mochilas, guaireñas". EO

\section{Consumidores por fidelización}

Se entiende por fidelización "el mantenimiento de relaciones a largo plazo con los clientes de la empresa, obteniendo una alta participación en sus compras". Desde la perspectiva del marketing actual, "implica el establecimiento de sólidos vínculos y el mantenimiento de relaciones a largo plazo con los clientes" (López, 2012). Este tipo de consumidores se relacionan más con los atributos inmateriales que puede ofrecer la tienda. En este caso lo que tiene valor no es la compra de un día, sino la referencia de muchas compras anteriores y de servicios que le ha ofrecido la tienda al cliente. Estos son los clientes que gastan más dinero y los que más recomiendan la tienda a sus amigos y conocidos, además el cliente por fidelización establece un vínculo que genera valor a la tienda Universitaria. 


\section{Ilustración 1. Consumidores por fidelización}

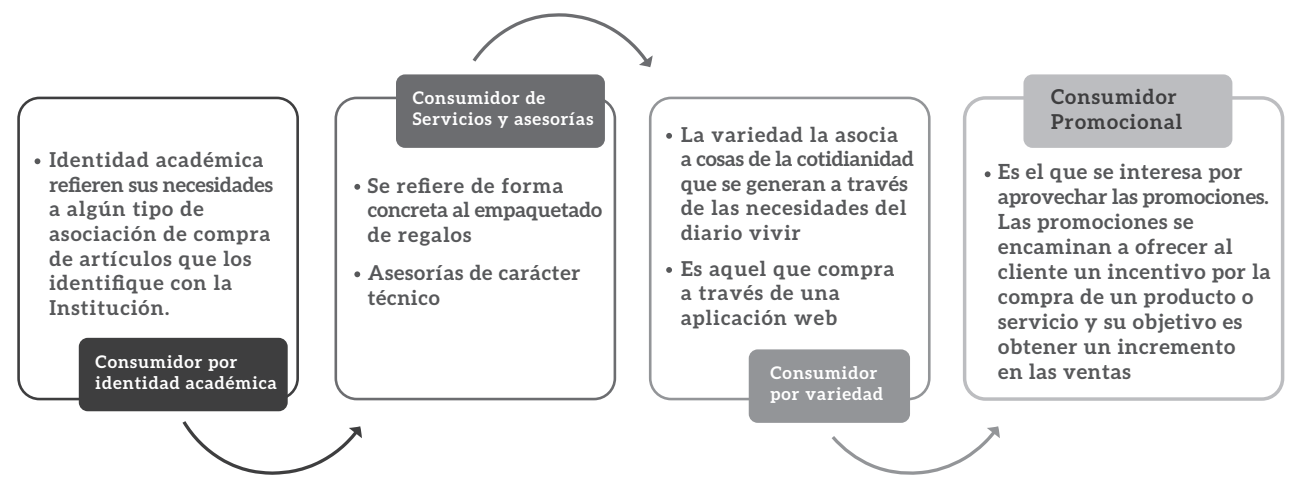

Fuente: Elaboración propia de los autores

\section{Consumidor por identidad académica.}

"La identidad universitaria está concebida, como el conjunto de dimensiones culturales compartidas por la comunidad universitaria, siendo la diversidad y la multiculturalidad los factores más importantes en el proceso de formación de la identidad institucional que viene a ser de suma importancia para el desarrollo de la persona dentro de la formación de su identidad académica" (Anumeth, 2012). La identidad académica se construye teniendo en cuenta diversos procesos de identificación que surgen de la institucionalidad o del orden social en el contexto universitario. El tipo de consumidores de identidad académica refieren sus necesidades a algún tipo de asociación de compra de artículos que los identifique con la universidad, a través de esta identidad, los consumidores orientan sus acciones y también le dan sentido a sus a sus prácticas cotidianas.

Como tienda universitaria debería ofrecer algo que me identifique. No sé. Bueno pienso dad es academia, primeramente. Y yo creo que sea complicado porque es un lugar que es de ventas y no tiene nada que ver... No sé. La verdad no sabría...E07

"Yo tenía hasta el carro con stiker. [...] Más de uno está orgulloso de estudiar acá. Yo sé que eso podría venderse. Y suelen hacerse como largos para que se pongan en la parte de atrás. Está muy de moda el desde... Como esa universidad era más vieja..." D03

\section{Consumidor por Variedad.}

En este tipo de consumidores, la variedad es una de las características que espera encontrar, asociando artículos que en su primer criterio no estaba dispuesto a comparar, la variedad la asocia a cosas de la cotidianidad que se generan a través de las necesidades del diario vivir.

"Depende de lo que me ofrezcan, yo estoy abierta a las posibilidades" E02

"De pronto cuando pase, y cuando me quedo aquí. A mí no me gusta comprar en 
las maquinitas, entonces ya sé qué voy a ir a comprar allá porque a mí me encanta el dulce y allí es donde hay productos de esos" E05

Asimismo, como opción el consumidor virtual de la tienda universitaria espera comprar a través de una aplicación web, que podría ser una tienda on line, para tener que romper los límites del espacio, aunque a su vez la tienda física la considera como un respaldo de su compra. Es como refiere a una tienda virtual, que para este tipo de consumidores tienen muchas facilidades para comercializar en variedad.

Según Cristóbal (2001) "Algunos autores afirman que para considerar un website como un establecimiento virtual debe cumplir el único requisito de habilitar la posibilidad de compra a través de cualquier medio Internet (desde los más complejos formularios de pedidos, hasta el simple correo electrónico), comprometiéndose a la entrega en el domicilio que indique el cliente"

"Si la tienda pudiera dar la opción de algún descuento de cualquier elemento de tipo virtual, podría entrar a la tienda" E08

Y también dice otro entrevistado:

"Estaría dispuesta a comprar on line. Porque no vas a..., simplemente te llega el paquete llega acá y hay una intermediación".E05

\section{Consumidor de Servicios y Asesorías:}

En el ámbito del marketing, se entiende por servicio un cúmulo de tareas desarrolladas por una compañía para satisfacer las exigencias de sus clientes (Definición De). De este modo, el servicio podría presentarse como un bien de carácter no material. Los consumidores de servicio y asesoría de la tienda universitaria se caracterizan por querer recibir sugerencias y formas de transformar y/o mejorar los productos que adquieren (sea en la tienda y/o fuera de ella), por ejemplo, el servicio de empaquetado de regalos, los clientes se muestran muy satisfechos de este servicio, algunos compran en un establecimiento diferente y se acercan a la tienda para solicitar el empaque por el que pagan.

“...sobre todo me gusta la parte de empaques. Si compro un libro en la tienda, voy allá para que me lo empaquen. [...] Si, tú me quitas la envoltura y quedo en el aire. La envoltura es bonita sí, con papel trasparente, moñito y vaina. Sobre todo que yo, en la época de cumpleaños de mi hijo, le regalo libros de la tienda". A03

Además, cuando el consumidor de la tienda universitaria se refiere a asesoría, genera la inquietud de comprar artículos relacionados con el ámbito de su profesión, elementos y/o productos tecnológicos, que le generarían la confianza de adquirirlos en un local de la institución.

“...Con respecto a las cámaras y demás artículos de que hablan los alumnos. Si estuvieran en la tienda, tal vez, pero deberían ir acompañados de una gran asesoría por el gran todo ahí tirado, a que entres a una tienda y cuando tú preguntes y la persona sepa específicamente responderte, si eso que vas a adquirir cumple con lo que tú requieres. [...] Es muy difícil para un estudiante, adquirir un aparato tecnológico tal como una. A10 


\section{Consumidor de Promocional:}

Este tipo de consumidores esperan la oportunidad de encontrar en la tienda universitaria productos que le generen la posibilidad de encontrarlos más económicos, sean porque existan convenios de los proveedores con la universidad y/o porque son puestos en márgenes más económicos por ser el mayor número de consumidores los estudiantes quien en su mayoría no poseen niveles de ingreso altos. Además, como señala Thompson, 2005 "la promoción es el conjunto de actividades, técnicas y métodos que se utilizan para lograr objetivos específicos, como informar, persuadir o recordar al público objetivo, acerca de los productos y/o servicios que se comercializan", para conocer el concepto promoción, el cual es base de las características asociadas al consumidor por variedad, donde el individuo espera encontrar muchos artículos y se interesa por aprovechar las promociones sobre estos mismos, con la posibilidad de hallar a ofrecer al cliente un incentivo por la compra de un producto o servicio y su objetivo es obtener un incremento en las ventas.

"Yo soy de las que revisa promociones por Instagram, y todas esas cosas. Entonces me parece que es un buen medio para comunicar" E04

\section{Consumidor por frecuencia.}

La frecuencia en este caso refiere a los tiempos de vista de un cliente: la tienda universitaria posee consumidores habituales y ocasionales.

Ilustración 3. Compradores por frecuencia

- Consumidor que visita la tienda casi que a diario.

- Se ha logrado crear una relación con la tienda.

- Algunos buscan productos y otros solo por darle continuidad a su relación con los empleados o la tienda misma.

\section{Consumidor} Habitual

\section{Comprador}

Ocasional
- Visita la tienda en contadas oportunidades. No hace recorridos por ella y se dirige puntualmente a lo que necesita para la ocasión. Solo esporádicamente compra el producto.

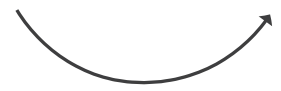

Fuente: Elaboración propia de los autores

\section{Consumidor Habitual:}

Este "es un consumidor que casi a diario mantiene contacto con su tienda preferida. Algunos lo hacen en busca de productos y otros por darle continuidad a su relación con el tendero o con sus amigos. Demanda respeto y trato personalizado y considera 
la fidelidad como un aspecto clave en sus relaciones comerciales" Páramo (2012). Los consumidores habituales ingresan a la tienda dos o tres veces por semana, sea para comprar, solo antojarse y/o mirar los artículos nuevos, y los que también aún permanecen.

A uno le gusta mucho el detalle, de pronto el novio, el amigo, fuera de los días exclusivos de Amor y Amistad a uno le gusta hacer eso de vez en cuando. E02

Tres veces por semana. E 05.

\section{Consumidor Ocasional:}

Sólo visita la tienda en contadas oportunidades. No hace recorridos por ella y se dirige puntualmente a lo que necesita para la ocasión, solo esporádicamente compra el producto. Es esta que señala:

La verdad no he visitado la tienda porque siento que sus costos son un poco elevados en comparación con el resto del mercado. [...] He entrado dos veces: A acompañar una amiga a comprar una tarjeta para el novio y para organizar un cumpleaños. E08

Igualmente, este tipo de consumidores tienen imaginarios de características que en alguna visita la tienda no agrado a sus gustos personales y los mantienen como premisa para no visitarla continuamente.

\section{CONCLUSIONES}

El estudio realizado con el apoyo antropológico y etnográfico, logro develar desde el comportamiento natural de los consumidores de una tienda universitaria unas categorías propias de la cultura, del consumo y de la misma condición específica del segmento de mercado estudiado. Además, categorías que no habían sido descubiertas y que impulsan a un desarrollo más acertado de la comercialización de productos y servicios que este tipo de entes económicos puede ofrecer.

En la tienda universitaria de esta reconocida institución universitaria se identificaron tres tipos de consumidores, que son: 1. Consumidores por línea, que a su vez comprende los consumidores de artículos personales, consumidores de comestibles y drogas, consumidores de productos típicos, consumidores por identidad académica, consumidores por variedades. 2. Consumidores por fidelidad, cuyo grupo comprende: consumidores de servicios y de asesorías, consumidor promocional, y consumidor virtual; 3. Consumidor por frecuencia, que comprende: consumidores habituales y consumidores ocasionales.

\section{AGRADECIMIENTOS}

Los autores desean agradecer la colaboración de Johnny Pava Cañas funcionario de la institución universitaria en la Ciudad de Barranquilla - Colombia. 


\section{REFERENCIAS BIBLIOGRAFICAS}

Anumeth (2012). Identidad Académica y Universitaria. Recuperado de: https:// anumeth.wordpress.com/2012/12/07/identidad-academica-y-universitaria/

Álvarez, C. (2014). Comunicación y Culturas de consumo. Escuela Latinoamericana. Recuperado de: http://alvarezteran.com.ar/wp-content/uploads/downloads/ 2014/03/Manual-Cultura-de-Consumo-2014.pdf

Caldentey, P. y Gómez, A. C. (1996). Productos típicos, territorio y competitividad, Agricultura y Sociedad, 80, 57-82. Recuperado de: https://dialnet.unirioja.es/servlet/ articulo?codigo $=83020$

CEPAL (2000): Juventud, población y desarrollo. Comisión Económica para América Latina y el Caribe. Santiago de Chile: CEPAL.

Cristóbal-Fransi, E. (2001). La tienda en Internet: cómo diseñar y dar a conocer un establecimiento virtual con éxito. Boletín económico de ICE, Información Comercial Española, 2001, 2687, 1001-1011. Recuperado de: https://repositori. udl.cat/handle/10459.1/330

Del Río-Cortina, J.L., Cardona-Arbeláez, D., \& Guacarí-Villalba, A. (2017). Responsabilidad social empresarial y construcción de la marca: una nueva mirada a las estrategias de gestión. Revista de Investigación, Desarrollo e Innovación, 8(1), 49-60.

Del-Rio, J., Cardona-Arbeláez, D., \& Simancas-Trujillo, R. (2017). Propuesta de un modelo teórico de branding para el posicionamiento de la marca universitaria. Revista Espacios, 38,53.

Galindo (1998). Técnicas de investigación en sociedad, cultura y comunicación. Addison Wesley Longman.

Gómez-Romo, M., López-Gomez, A., \& Carvajal-Larenas, R. (2017). La Responsabilidad Social en las PyMEs del Sector Calzado en Ecuador. Panorama Económico, 25(2), 195-208. DOI: https://doi.org/10.32997/2463-0470-vol.25-num. 2-2017-2074

Hernández, C. y Viveros, C. (2012). Fundamentos de Marketing. Pearson Education.

Fierro, I., Cardona, D. A., \& Gavilanez, J. (2017). Digital marketing: a new tool for international education. Pensamiento \& Gestión, (43), 241-260.

Jaimovich, D. (2015). Missing links, missing markets: Evidence of the transformation process in the economic networks of Gambian villages. World Development, $66,645-664$.

López, L. M. (2012). La tributación en el IVA de los programas de fidelización de los clientes: la STJUE de 7 de octubre de 2010. Noticias de la Unión Europea, (330), 121-126. 
Passos-Simancas, E., \& Arias-Aragonés, F. (2016). El capital humano como factor de competitividad en la industria hotelera y turística en Cartagena. Panorama Económico, 24, 269 - 282. DOI: https://doi.org/10.32997/ 2463-0470-vol.24-num.0-2016-1561

Martínez Díaz, D. (2009). Valores y creencias en el consumo de comida en los hogares barranquilleros. Pensamiento y Gestión, 27 (dic. 2009).

McCracken, G. (1990). Cultura y comportamiento de consumo: Una perspectiva antropológica. Journal of the Market Research Society, 32 (1) 3-11.

Páramo Morales, D. (2004). El fenómeno de consumo y el consumo en marketing. Convergencia Revista de Ciencias Sociales, (34).

Páramo Morales, D., Martínez Díaz, D., \& Ramírez Plazas, E. (2007). Cultura de consumo de bebidas alcohólicas (1st ed.). Bogotá: Universidad Surcolombiana.

Páramo, Contreras, Arenas (2010). Canal Tradicional en Barranquilla: Evolución y prácticas comerciales. Libro. Ediciones ASABA. Barranquilla.

Páramo Morales, D. (2012). Tiendas de barrio en Colombia. Pensamiento y Gestión, (32).

Salazar-Mosquera, G. (2017). Factores determinantes del desempeño financiero en el sector manufacturero en la República del Ecuador. Panorama Económico, 25(2), 243-254. DOI: https://doi.org/10.32997/2463-0470-vol.25-num.22017-2076

Schouten, J. \& McAlexander, J. (1995). Subcultures of Consumption: An Ethnographic of the New Bikers. Journal of Consumer Research, 22, 43-61.

Serrano, F. y Goyeneche, D. (2008). Creación de una Tienda Universitaria con productos marca UIS. Bucaramanga, Universidad Industrial de Santander. 